\title{
Correction to: Digital Signal Processing with Field Programmable Gate Arrays
}

\section{Correction to:}

U. Meyer-Baese, Digital Signal Processing with Field Programmable Gate Arrays, Signals and Communication Technology, https://doi.org/10.1007/978-3-642-45309-0

The original version of this book was inadvertently published without the Electronic Supplementary Materials information (CD-ROM......) on the copyright page, which has now been included. The book has been updated with the change. 